\title{
Synthesis of Solanoeclepin A
}

\section{Key words}

solanoeclepin A

Meinwald rearrangement

stereoselective epoxidation

Charette-modified Simmons-Smith cyclopropanation

intramolecular Diels-Alder reaction

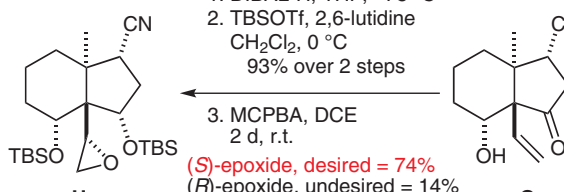
N TMSOTf, 2,6-lutidin

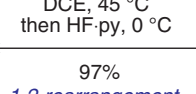<smiles>C=C[C@]1(O)C[C@H](C#N)[C@]2(C)CCC[C@@H]3O[C@]321</smiles>
tert-butyl hydroperoxide

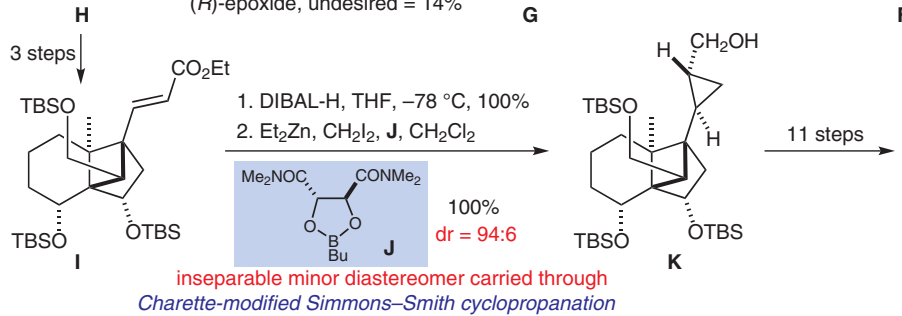
R) -epoxide, undesired $=14 \%$ G

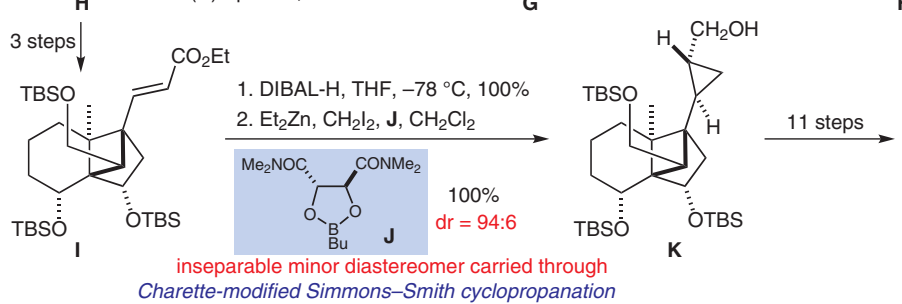

$\mathbf{F}$ directed
stereoselective
epoxidation

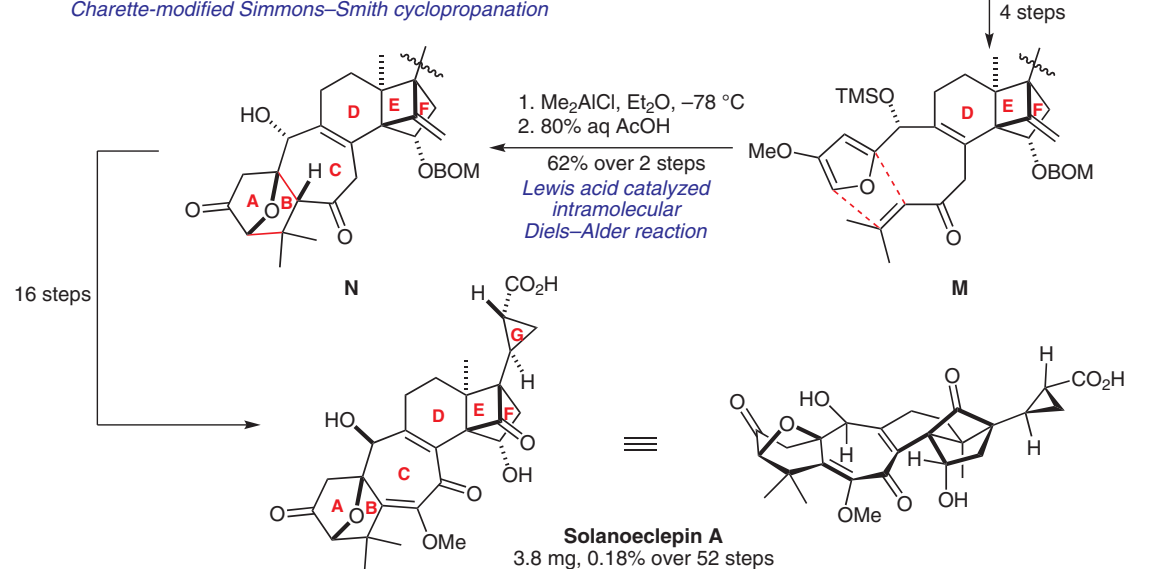

Significance: Solanoeclepin A, a complex triterpenoid, was isolated in 1986 by Mulder and coworkers and is a hatch stimulus for the potato cyst nematode, which inflicts considerable damage to crop plants. Cyst nematodes are notoriously hard to exterminate and as such attention has been drawn to study of such stimuli in order to eradicate this problem.
Comment: Key steps in the synthesis include several rearrangements and a Charette-modified Simmons-Smith cyclopropanation to complete the key DEFG fragment $\mathbf{L}$. Further elaboration via an intramolecular Diels-Alder reaction completed the ABCDEFG scaffold (N). The first total synthesis of solanoeclepin A was completed in further 16 steps. 